\title{
Response of Actively Cooled Metal Foam Sandwich Panels Exposed to Thermal Loading
}

\author{
Jeseph F. Rakow* and Anthony M. Waas $\ddagger$ \\ University of Michigan, Ann Arbor, Michigan 48109
}

DOI: $\underline{10.2514 / 1.24813}$

\begin{abstract}
In this paper, experimental and analytical results for the thermomechanical response of actively cooled metal foam sandwich panels are presented. With a favorable strength-to-weight ratio and a high specific internal surface area, sandwich panels with metal foam cores are proposed for actively cooled load-bearing components in aerospace thermal protection system applications. First, inconel foam sandwich panels are subjected, via experimental means, to through-the-thickness thermal gradients, defined by fixed temperature conditions on opposite face sheets. With clamped boundary conditions, provided through a test fixture designed to minimize the common conflict of thermal and mechanical boundary conditions in experimental apparatus, the metal foam sandwich panels exhibit out-ofplane bending deformation. The thermomechanical deformation within the panel is then mitigated through active cooling, achieved by pumping compressed air through the metal foam core. Subsequently, the experimental measurements are used to develop a sequentially coupled thermomechanical finite element model. Central to the numerical characterization of metal foam sandwich panels is an understanding of the response of metal foam under shear loading. The material model for the foam is taken from a series of experimental measurements of the shear response of metal foam, providing density-dependent relationships for material stiffness and strength. The numerical model provides a strain-temperature history for metal foam sandwich panels under through-thethickness thermal gradients. The results are shown to agree with the experimental measurements.
\end{abstract}

\section{Introduction}

$\mathbf{T}$ HERMAL protection during high-speed flight is one of the most pressing issues in the advancement of hypersonic vehicles. Since the development of the $X-2$ and $X-15$ vehicles, designed to capitalize on the past supersonic accomplishment of the $X-1$, the technology to propel vehicles has been arguably more mature and more successful than the technology to protect vehicles from the resulting aerodynamic heat loads, as marked by the disintegration of the space shuttle Columbia during hypersonic reentry. This limitation has manifested and dominated a variety of aspects of hypersonic vehicle operation, from flight planning to vehicle maintenance to flight performance envelopes, examples of each follow. Regarding flight planning, the ceramic thermal protection system (TPS) on the space shuttle cannot fly in wet weather because its tiles would likely be damaged by impact from precipitation. Regarding vehicle maintenance, the TPS on the space shuttle, as a whole, dominates the maintenance requirements of the vehicle, demanding 32,000 of the 50,000 man-hours of inspection and repair between flights. Regarding performance envelopes, on its recordsetting flight above Mach 6 components of the $X-15$ were virtually destroyed when shock-shock interactions pierced holes in the inconel airframe, a heat sink TPS. As illustrated by these examples, the current state-of-the-art in TPS technology cannot efficiently manage the demanding requirements of hypersonic vehicle operation.

One concept for advanced structural thermal protection systems, and one that promises attractive thermal load management [1], is an actively cooled thermal protection system in which load-bearing structural members are integrated with coolant passages. Unlike the dual-component ablative TPS onboard the manned capsules of the Mercury, Gemini, and Apollo flight vehicles and unlike the dual-

Received 25 April 2006; revision received 29 August 2006; accepted for publication 29 August 2006. Copyright (C) 2006 by Anthony M. Waas. Published by the American Institute of Aeronautics and Astronautics, Inc., with permission. Copies of this paper may be made for personal or internal use, on condition that the copier pay the $\$ 10.00$ per-copy fee to the Copyright Clearance Center, Inc., 222 Rosewood Drive, Danvers, MA 01923; include the code $\$ 10.00$ in correspondence with the CCC.

${ }^{*}$ Graduate Student Research Assistant, Aerospace Engineering Department.

†Professor, Aerospace Engineering Department; dcw@umich.edu. Author to whom all correspondence should be addressed. Associate Fellow AIAA. component insulated system employed on the space shuttle, a more advanced structural thermal protection system may be a singlecomponent multifunctional structure that bears both mechanical and thermal loads.

To this point in time, however, true integration and multifunctionality have not been achieved. An example of a firstgeneration actively cooled structural panel is shown in Fig. 1 [2]. This panel is a standard honeycomb-cored sandwich panel that has been modified with a series of coolant tubes on the inside of the external face sheet. The problems associated with this type of construction are readily apparent:

1) The coolant tubes interrupt the path for shear load transfer between the face sheets and the honeycomb core.

2) Machining of the honeycomb and the lay-up of the panel to accommodate these tubes create significant manufacturing difficulties.

3) The discrete nature of the coolant passages causes severe thermal-stress gradients at locations throughout the panel.

4) Blockage in discretized coolant passages leads to local hot spots of reduced strength in the load-bearing structure.

Actively cooled metal foam sandwich panels (MFSPs) promise a truly multifunctional structure for the management of both thermal and mechanical loads. The multifunctionality of cellular metals is addressed in [3]. An actively cooled MFSP is a sandwich panel with an open-cell metal foam core that is integrally bonded (i.e., brazed) to metal face sheets. While the panel bears airframe flight loads, aerodynamic heat on the panel's outer surface is conducted through the outer face sheet and, with the assistance of the conductive foam, into coolant passing through the core of the panel.

Metal foam, which bears mechanical shear loads within the MFSP while conductively distributing heat from the outer surface of the panel into and throughout the cross-sectional flow of the coolant, is a critical component of the multifunctional panel. In its open-cell form (Fig. 2), metal foam has a substructure similar to foam found in seat cushions and packing materials, but the substructure is made of a metallic alloy (i.e., aluminum, titanium, inconel, and copper).

Metal foam is central to the improvements offered by MFSPs over previous actively cooled panel concepts:

1) The foam and face sheets provide a monolithic single-material structure with no inherent intrastructural mismatch of coefficients of thermal expansion. 


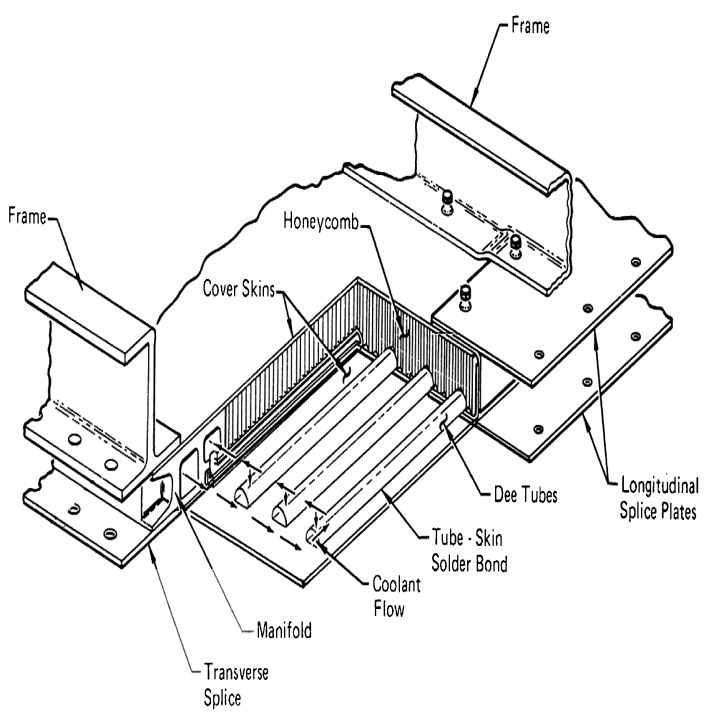

Fig. 1 A typical first-generation actively cooled panel concept [2].

2) Through-the-thickness shear response and heat transfer can be controlled by controlling the foam density [4-6].

3) The metal foam core provides a network of nondiscreet coolant passages, eliminating the severity of the effects of local coolant blockage.

4) With high internal surface area per unit volume, $\sim 1800 \mathrm{~m}^{2} / \mathrm{m}^{3}$ $\left(\sim 549 \mathrm{ft}^{2} / \mathrm{ft}^{3}\right)$, metal foam facilitates extremely high structurecoolant interaction per unit volume.

5) In addition to conducting heat, the foam core improves the convective heat transfer capability of the system by creating turbulence in the coolant, even at low Reynolds numbers. The departure from laminar flow introduces a nonzero eddy coefficient, $\epsilon_{H}$, into the boundary layer equations for steady incompressible flow with constant properties, which increases the rate of heat flux, $Q$, to the coolant relative to what would result from laminar flow by the amount

$$
\boldsymbol{Q}=-\rho \mathbf{c}_{p}\left(\alpha^{*}+\epsilon_{\mathbf{H}}\right) \frac{\partial \overline{\mathbf{T}}}{\partial \mathbf{z}}
$$

In (1), $\rho, c_{p}$, and $\alpha^{*}$ are the density, specific heat at constant pressure, and thermal diffusivity of the coolant, respectively, and $\partial \bar{T} / \partial z$ is the time-averaged thermal gradient field perpendicular to the flow [7].

A critical factor in the ability of MFSPs to withstand thermal and mechanical flight loads is its ability to carry shear loads. In previous studies, the present authors have demonstrated and characterized the response of metal foams under shear loading through experimentation [4], numerical simulation [5], and a micromechanics-based analysis [6]. The purpose of the present work is to study the thermomechanical response of an actively cooled MFSP that is subjected to through-the-thickness thermal gradients.

The present work demonstrates and analyzes a fully functioning prototype actively cooled MFSP. The core of this work is a thermostructural experiment and a finite element analysis of an MFSP with inconel face sheets and an inconel foam core subjected to active cooling with compressed air. (Inconel, selected for structural integrity under high heat flux and high structural temperatures, has served as a primary material for structural components on previous hypersonic aircraft such as the $X-15$.) The foam was supplied by ERG Aerospace (Oakland, California). The foam was brazed to the inconel sheets by Protomatic (Dexter, Michigan). For the inconel, the ultimate strength at room temperature is $500 \mathrm{MPa}$, modulus is $200 \mathrm{GPa}$, and Poisson's ratio is 0.29 . In the experiments and in the numerical model of the present work, the specimen is subjected to through-the-thickness thermal gradients under mechanical constraints, and the response of the panel is measured. Subsequently, the potential for control of this response through active cooling is demonstrated.

\section{Experimental Investigation}

\section{A. Procedure}

The experimental test article is shown in Fig. 3. The MFSP is a square panel, measuring $292 \mathrm{~mm}^{2}$ (11.5 in. $\left.{ }^{2}\right)$, composed of two inconel 625 face sheets, $1 \mathrm{~mm}(0.04$ in.) thick, with an inconel 625 foam core that has a thickness of $6.4 \mathrm{~mm}(0.25 \mathrm{in}$.), a relative density of $\rho_{f}=8 \%$ (the relative density is the density of the foam divided by the density of the parent material of the foam and is expressed as a percentage), and 20 pores/in. Also shown in Fig. 3 is the cooling manifold that is used to deliver the cooling air to the core of the MFSP. Figure 4 shows the test article and the cooling manifold mounted in a clamping frame made of $6 \mathrm{Al} 4 \mathrm{~V}$ titanium. Titanium is used for the frame because the frame must have a lower coefficient of thermal expansion than the test article to ensure contact between the frame and the MFSP throughout the experiment. The frame has two
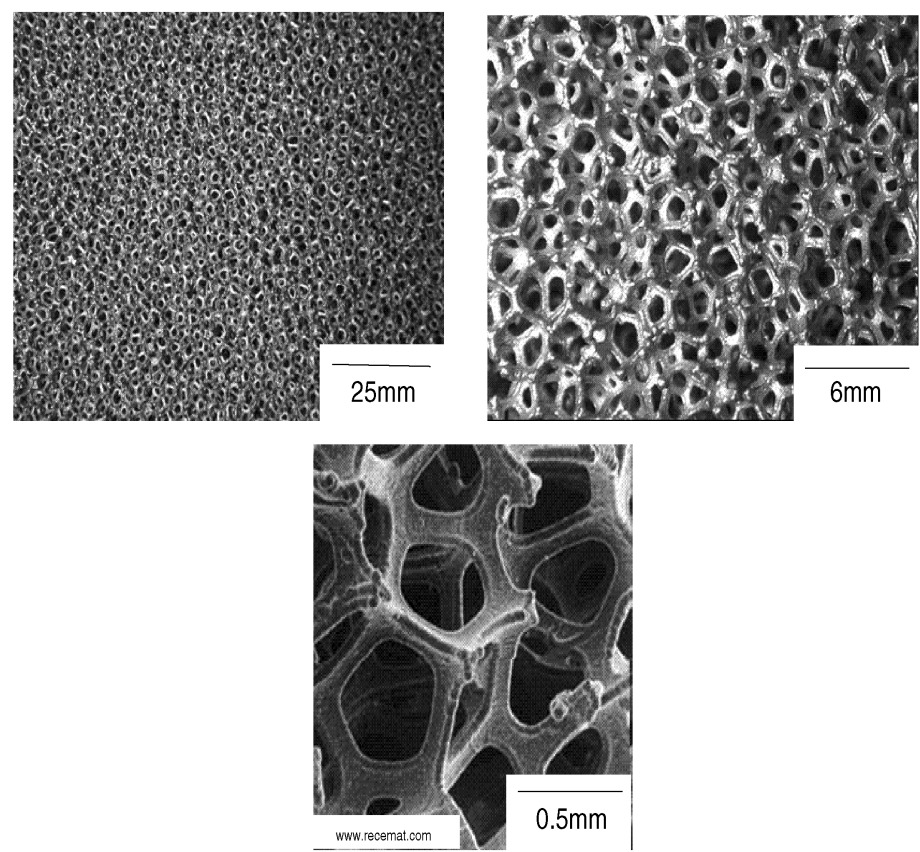

Fig. 2 Open-cell metal foams. 


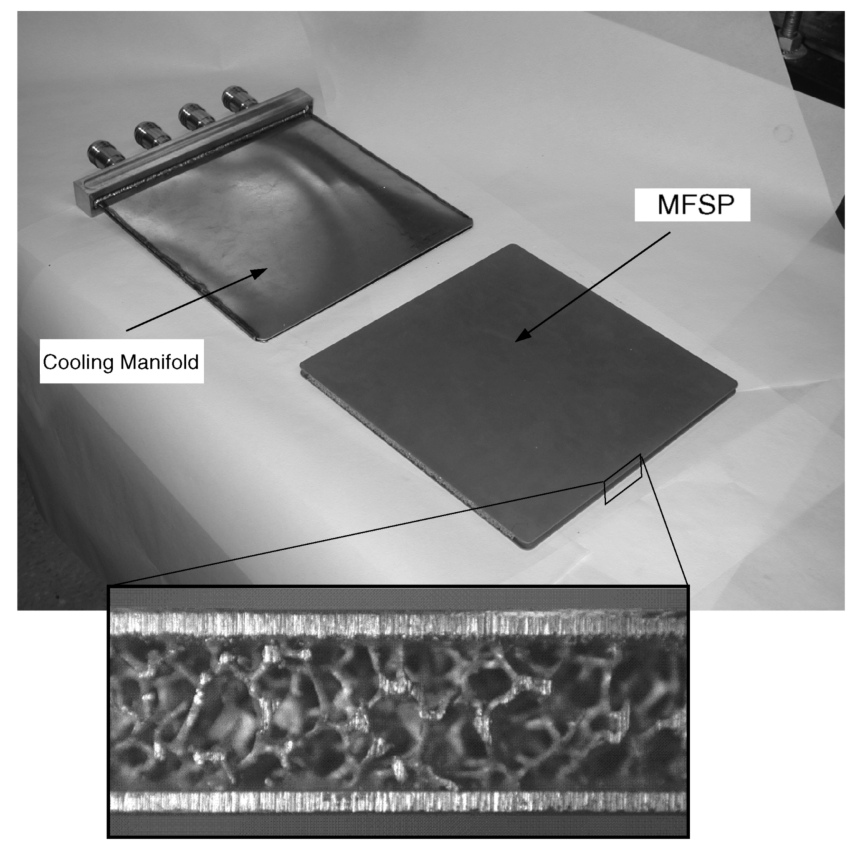

Fig. 3 The experimental cooling manifold and MFSP.

identical pieces that are clamped together by the platens of the heat press to provide a clamped boundary condition along the border of the MFSP. This configuration creates a central square test section measuring $216 \mathrm{~mm}^{2}\left(8.5 \mathrm{in}^{2}\right)$. The frame also provides a leak-proof seal for the coolant flowing through the MFSP. Additional benefits of this clamping frame, particularly with respect to experimental thermomechanical boundary conditions, are discussed in detail by the present authors in previous publications [ $[\underline{8}, \underline{9}]$.

The frame-panel assembly is subjected to thermal loading in a dual-platen heat press for which the temperature of each platen is programmable and controlled independently. A diagram of the panel-frame assembly in the heat press is shown in Fig. 5. The top platen (labeled "hot platen" in Fig. 5) provides heating to the test article. The platen does not allow control of the details of the time-dependent temperature history such as that encountered by the access to space reference vehicle [10]. The use of quartz heat lamps provides for this provision and such a test facility is currently being constructed. The temperature of the top platen is set to one of several

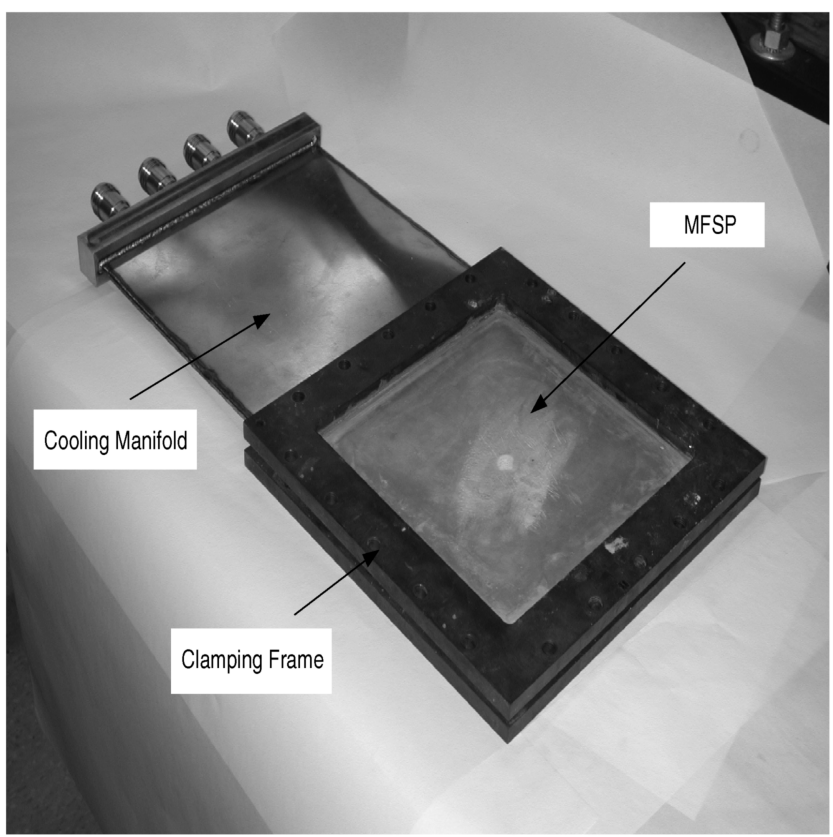

Fig. 4 MFSP in the clamping frame with the cooling manifold attached.

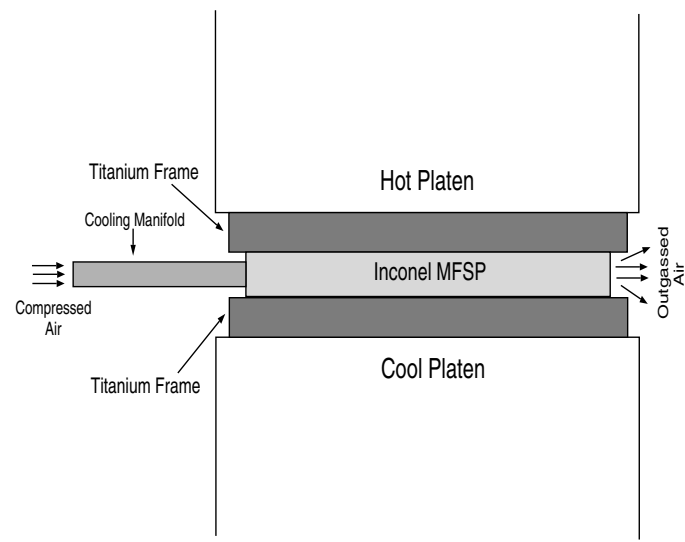

Fig. 5 Schematic of the experimental setup for thermal loading of MFSPs.

predetermined temperatures, as addressed below. The temperature of the bottom platen (labeled "cool platen") is uncontrolled, but the platen provides sufficient thermal inertia for the bottom surface of the panel to remain at its initial room temperature throughout the experiment. These conditions on the bottom surface of the test article simulate the desired conditions on the interior of a high-speed air vehicle. For the remainder of this discussion, the surface of the panelframe assembly subjected to direct heating from the hot platen will be called the exterior, as in the exterior of the vehicle; the surface of the panel-frame assembly not subjected to direct heating will be called the interior, as in the interior of the vehicle.

A sample thermal loading profile used in the experiments is shown in Fig. 6. At point A, the MFSP, the clamping frame, and the heat press are each at room temperature. Between point $A$ and point $B$, the hot platen of the heat press is heated until it reaches a predefined temperature. During this time, the panel-frame assembly is intentionally insulated from the hot platen so that it remains at room temperature. Once the target temperature is reached on the hot platen, the exterior surface of the frame is put in contact with the hot platen of the heat press, while the interior surface remains in contact with the cool platen. This occurs at point $\mathrm{B}$. Between point $\mathrm{B}$ and point $\mathrm{C}$, a dynamic thermal gradient develops through the thickness of the MFSP, and temperatures throughout the panel increase until thermal equilibrium is established, which occurs at point $\mathrm{C}$. The data collected between point $\mathrm{B}$ and point $\mathrm{C}$ represent the thermomechanical response of an uncooled MFSP to through-thethickness thermal gradients. Note that the equilibrium temperature of the exterior surface of the MFSP and of the exterior surface of the

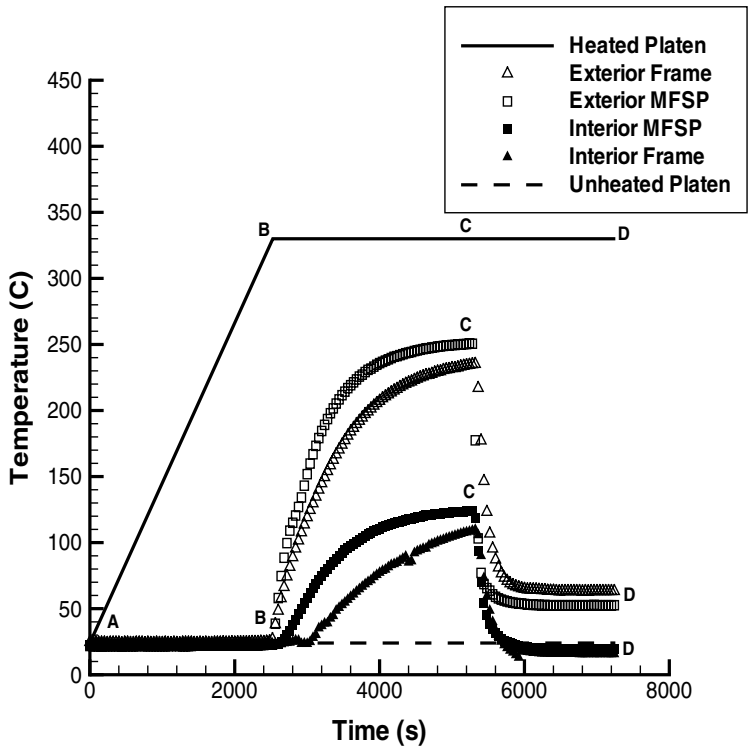

Fig. 6 Sample experimental temperature history. 
frame is less than the temperature of the hot platen. This is attributed to the fact that the experimental setup is not insulated, heat is allowed to escape to the atmosphere. At point $\mathrm{C}$, the cooling is activated, causing the temperature of the assembly to drop, reaching a new thermal equilibrium (point D) associated with the actively cooled MFSP. At point D, the experiment is terminated. The thermomechanical response of the MFSP with active cooling, compared with that without cooling, demonstrates the effectiveness of the actively cooled MFSP in managing the heat transfer through the panel and, therefore, the thermostructural deformation within the panel.

Three experiments are conducted, each at a different fixed temperature for the hot platen: $260^{\circ} \mathrm{C}, 330^{\circ} \mathrm{C}$, and $540^{\circ} \mathrm{C}\left(500^{\circ} \mathrm{F}\right.$, $626^{\circ} \mathrm{F}$, and $1004^{\circ} \mathrm{F}$ ). The hot platen temperatures are chosen based on the limitations of the strain gauges as well as the heat press. The maximum service temperature of the strain gauges is near $330^{\circ} \mathrm{C}$ $\left(626^{\circ} \mathrm{F}\right)$, and so the first two temperatures explore the range of measurable structural deformations. The third temperature, $540^{\circ} \mathrm{C}$ $\left(1004^{\circ} \mathrm{F}\right)$, is the upper temperature limit of the heat press. Under the condition of $540^{\circ} \mathrm{C}\left(1004^{\circ} \mathrm{F}\right)$, the strain gauges on the exterior surface of the test article will overheat and be destroyed. However, the gauges on the interior surface of the panel will not overheat before the experiment is complete. Therefore, valuable information may be captured on the interior surface of the test article at this elevated temperature.

The test article is fitted with a manifold to allow for compressed air to be passed through the core of the MFSP. The compressed air is maintained at room temperature under $6.9 \mathrm{MPa}$ (1000 psi). The flow rate and the gauge pressure of the air passed through the panel are regulated to be constant for each experiment: $0.85 \mathrm{~m}^{3} / \mathrm{min}$ $\left(30 \mathrm{ft}^{3} / \mathrm{min}\right)$ and $69 \mathrm{kPa}(10 \mathrm{psi})$. Once through the panel, the air is simply out-gassed to the laboratory surroundings. The parameters of the coolant flow are selected based on the experimental investigation presented in the appendix of the present paper. Within that work, two parameters are studied with respect to the thermal performance of the actively cooled MFSP: 1) the effect of foam core density and 2) the effect of pressure drop and, therefore, flow rate. In each of the studies, MFSPs are subjected to a through-the-thickness thermal gradient and are cooled with compressed air. The results provide an indication of the airflow rates required for substantial cooling of the MFSP's analyzed in the present work.

The panel-frame assembly is instrumented with pairs of back-toback high-temperature fully encapsulated Karma-based alloy strain gauges and type-K Nextel-insulated thermocouples in the locations depicted in Fig. 7. The strain gauges provide in-plane deformation measurements, and the back-to-back pairs provide an indication of out-of-plane deformation. The thermocouples provide temperature histories for the MFSP and for the frame that are used as the thermal loading input for the finite element analysis presented below in Sec. III.

\section{B. Results and Discussion}

Figure $\underline{8}$ shows the response of the inconel MFSP when subjected to a hot platen temperature of $330^{\circ} \mathrm{C}\left(626^{\circ} \mathrm{F}\right)$ and then actively cooled. The strain data presented in the plot is the total strain (thermal and mechanical), which has been obtained via standard techniques for measuring thermomechanical strains with foil strain gauges [11]. The initial plateau (point A to point B) represents the time before the panel is exposed to the hot platen. Once exposed, at point B, a strain response rapidly develops on both the hot and cool surfaces of the panel. This strain is a combination of tensile thermal strain and compressive mechanical strain due to the titanium frame. As is clear from the plots, the interior and exterior surfaces of the MFSP have qualitatively similar responses at different magnitudes.

As the time of exposure to thermal loading increases, thermal equilibrium is reached throughout the panel, which translates to mechanical equilibrium demonstrated by the plateau response at point $\mathrm{C}$. The difference between the strain levels on the interior and exterior sides of the panel throughout the response indicates a combination of thermal bending and of thermal shearing of the panel.

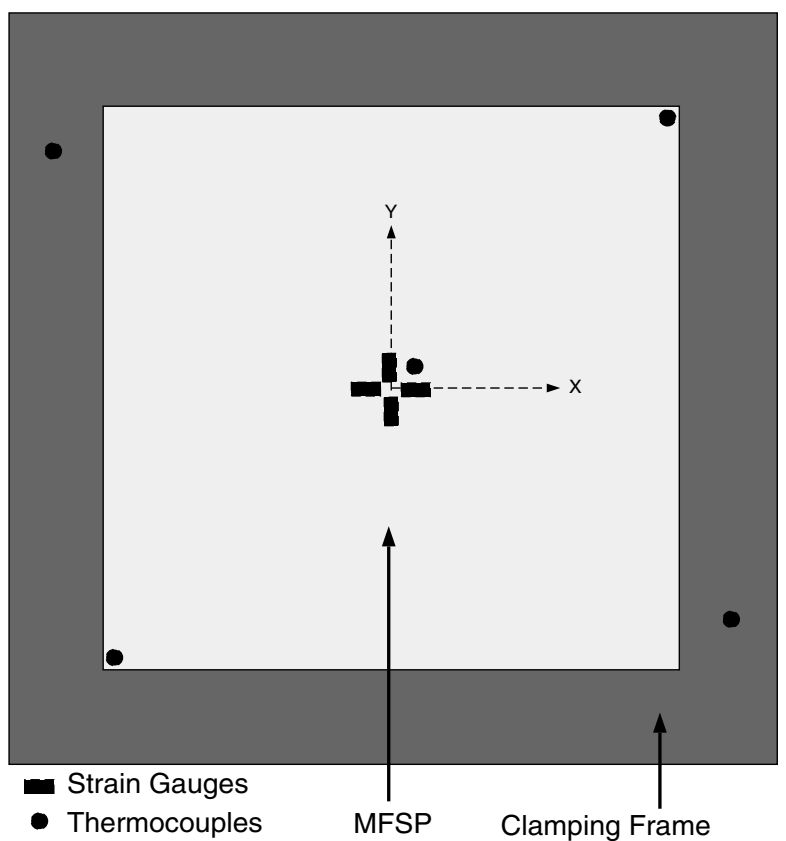

Fig. 7 Locations of the strain gauges and thermocouples employed in the experiments.

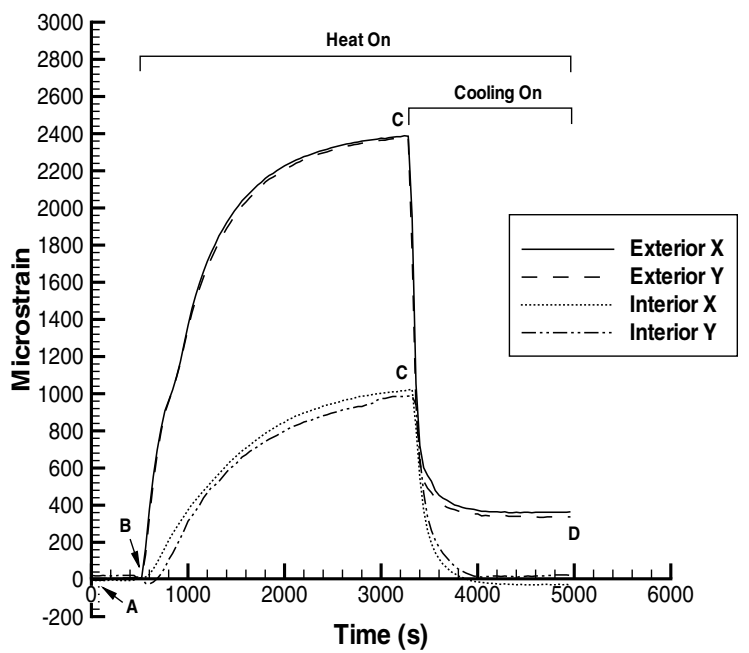

Fig. 8 Response of the MFSP with a platen temperature of $330^{\circ} \mathrm{C}$ $\left(626^{\circ} \mathrm{F}\right)$.

The magnitude of the total strain increases with time until the steady state is reached. This initial response curve indicates the response of the inconel MFSP without any active cooling.

After the plateau response has been reached, the cooling is activated at point $\mathrm{C}$, and the strain levels on both the interior and exterior sides of the panel drop dramatically. In fact, point D in Fig. 8 indicates that, by way of active cooling, the strain level on the interior side of the panel is brought back to its initial unheated configuration even though the specimen is still exposed to the fixed temperature of the hot platen. The data in Fig. 8 demonstrate that active cooling can effectively manage the temperature and deformation on the interior side of the panel such that the effects of external heating are no longer present.

Figure 9 shows the response of the inconel MFSP when the exterior side of the panel is subjected to a platen temperature of $540^{\circ} \mathrm{C}\left(1004^{\circ} \mathrm{F}\right)$. As in the first two experiments, the response of the panel without active cooling (point $\mathrm{B}$ to point $\mathrm{C}$ ) includes increasing strain on each surface of the MFSP until equilibrium is established (point C). Within this response, Fig. 9 indicates the point at which the strain gauges on the exterior side of the panel are destroyed. Once the 


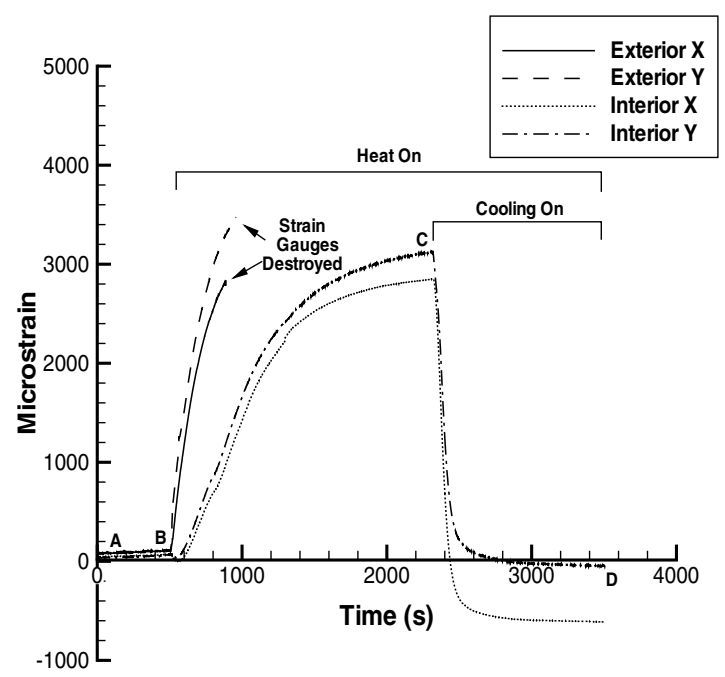

Fig. 9 Response of the MFSP with a platen temperature of $540^{\circ} \mathrm{C}$ $\left(1004^{\circ} \mathrm{F}\right)$.

uncooled MFSP reaches thermal equilibrium at point $\mathrm{C}$, the cooling is activated, and the response of the panel changes dramatically, eventually reaching a new equilibrium configuration at point $\mathrm{D}$. Again, even with a platen temperature of $540^{\circ} \mathrm{C}\left(1004^{\circ} \mathrm{F}\right)$, the structural response of the cool side of the panel can be controlled through active cooling such that the effects of external heating are no longer present.

These results are promising. Certainly, at some external temperature greater than $540^{\circ} \mathrm{C}\left(1004^{\circ} \mathrm{F}\right)$, the temperature of the interior side (i.e., the payload side) of the actively cooled MFSP will rise above room temperature and that side of the panel will exhibit an elevated thermomechanical response. In this case, the flow rate of compressed air could also be increased. The behavior of these panels when exposed to higher external temperatures, akin to those experienced in extreme hypersonic environments, is the subject of ongoing research.

One interesting aspect of the response curves shown in Figs. 8 and $\underline{9}$ is that the structural response is dramatically affected by the active cooling. To demonstrate the potential for attenuation of deformation within this system, the results of a series of experiments are presented. In these experiments, the uncooled panel is heated and the rapid initial response of the MFSP is measured, identical to the first set of experiments. With the temperature of the hot platen constant throughout the experiment and before reaching thermal equilibrium, the cooling is activated and the magnitude of the strain response decreases, as before. The cooling air is cycled on and off, the strain levels fluctuate accordingly, and an element of control of the

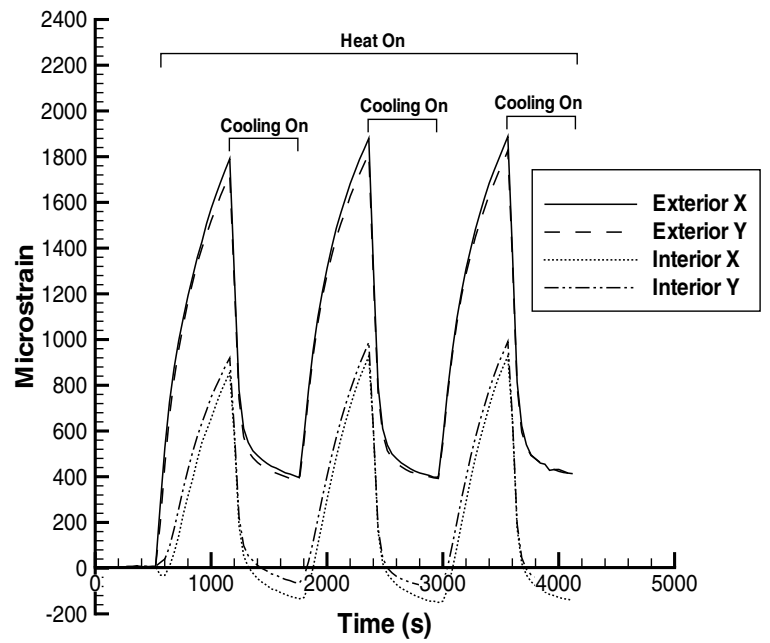

Fig. 10 Demonstration of attenuation of deformation with a platen temperature of $330^{\circ} \mathrm{C}\left(626^{\circ} \mathrm{F}\right)$. structural deformation, through active cooling, is demonstrated. The response curve for one of the experiments is presented in Fig. 10 for exposure to temperature of $330^{\circ} \mathrm{C}\left(626^{\circ} \mathrm{F}\right)$, respectively.

\section{Numerical Investigation}

In this section, a finite element model of the experimental panelframe assembly is developed and analyzed. Once validated with the existing experimental data, the model provides a tool for investigating critical design parameters such as materials selection and geometry, including foam density. In addition, and more immediately relevant, the model allows for further investigation into the dominant deformation mechanisms that produced the experimental results observed in Sec. II. The measured temperature histories of the MFSP and of the clamping frame (Fig. 6), captured by the thermocouples in the experiments described in Sec. II, are entered into the finite element model to serve as the thermal loading. These temperature histories are captured from multiple points on the MFSP and on the frame (see Fig. 7). The measured experimental temperature data is directly entere $\bar{d}$ into the finite element input file in tabular form. The strain response of the MFSP to this thermal loading, as predicted by the analysis, is then compared with the response measured experimentally and presented in Fig. $\underline{8}$ and $\underline{9}$.

\section{A. Geometry, Mesh, Boundary Conditions, and Loading}

The experimental load frame has two planes of symmetry with respect to geometry, boundary conditions, loading, and modes of deformation throughout the experiment. This allows for the finite element model to consist of a quarter of the panel-frame assembly. Both the MFSP and the frame are modeled entirely with threedimensional linear brick elements (C3D8), using the commercial finite element code ABAQUS [12]. The solution is obtained through a sequentially coupled thermal-stress solution algorithm in which the heat transfer problem is solved first providing nodal temperatures for each node in the model. These temperatures are then used for thermal loading in the solution of the mechanical problem. The model has approximately 36,000 elements and 128,000 degrees of freedom.

The MFSP is modeled as a solid partitioned into three regions: two for the face sheets and one for the core. The core and the face sheets of the MFSP each have three elements through the thickness. The foam core is modeled as a homogeneous isotropic solid that has effective temperature-dependent properties based on experimental measurements conducted by the present authors [4,5]. Detailed discussion of this and other material issues are addressed in the following section.

Displacement boundary conditions for all nodes on each plane of symmetry are such that

$$
u_{n}=0
$$

in which $u_{n}$ is the component of displacement normal to the plane of symmetry. The connection between the MFSP and the frame is modeled with a series of tie contacts, for which the frame nodes are the master nodes and the MFSP nodes are the slave nodes. These tie contacts represent perfect bonding such that

$$
\boldsymbol{u}_{\text {panel }}=\boldsymbol{u}_{\text {frame }}
$$

along the entire interface between the panel and the frame. In addition, with $z$ taken to be the direction perpendicular to the surface of the MFSP,

$$
u_{z}=0
$$

along the interface between the clamping frame and the heat press platens.

\section{B. Material Properties}

The face sheets are made of inconel 625. Temperature-dependent stress-strain curves for inconel 625, including the elastic and plastic regimes, are available from material databases [13]. The linear elastic response of inconel 625 does not change significantly within the temperature range of the experiments in the present work, $24-330^{\circ} \mathrm{C}$ $\left(75-626^{\circ} \mathrm{F}\right)$. In the model, the elastic properties are assumed to be 
independent of temperature over the temperature range to be modeled. Likewise, plastic deformation is not modeled in the face sheets because the measured experimental strains did not detect the presence of plastic deformation. Experimental measurements were obtained for the coefficient of thermal expansion (CTE) of the inconel face sheets and of the titanium frame over the range of temperatures encountered in the present experiment. These values are found to be $\alpha_{\text {inconel }}=12.8 \mu \epsilon /{ }^{\circ} \mathrm{C}\left(7.1 \mu \epsilon /{ }^{\circ} \mathrm{F}\right)$ and $\alpha_{\mathrm{Ti}}=$ $9.0 \mu \epsilon /{ }^{\circ} \mathrm{C}\left(5.0 \mu \epsilon /{ }^{\circ} \mathrm{F}\right)$ and are constant over the temperature range encountered in the present experiments. This information is used for the CTE of inconel and titanium in the simulation.

The foam core is also made from inconel 625. The core has 20 pores/in. and a relative density of $\rho_{f}=8 \%$. Elastic properties for the inconel foam in the model are scaled from elastic properties that have been determined experimentally by the present authors for aluminum foam of like construction $[\underline{4}, \underline{8}, \underline{9}]$. Appropriate scaling relations take on the following form:

$$
\frac{E_{\text {inconel }}^{\text {foam }}}{E_{\text {aluminum }}^{\text {foam }}}=\frac{E_{\text {inconel }}^{\text {solid }}}{E_{\text {aluminum }}^{\text {solid }}}
$$

The inconel foam core is assumed to remain elastic throughout the thermal loading history in the experiment and in the model. In addition, the inconel foam core is assumed to have the same CTE as the inconel face sheets, which is a commonly accepted assumption for metal foam [14].

\section{Results and Discussion}

Figure 11 shows the experimental data and the results of the finite element simulation. The plots indicate good agreement with the experiments. The favorable nature of the agreement may be attributed, in part, to the fact that, although a large portion of the strain magnitude is due to thermal expansion, the simulated thermal loading in the MFSP and in the frame is defined by the temperature history measured experimentally. The strain due to thermal expansion is governed by the CTE of the material and the temperature change, both of which have been included in the model, a priori, based on experimental measurements. Nonetheless, the simulation clearly captures the thermomechanical deformation measured by the strain gauges.

With agreement established between the finite element model and the experiments, the results of Fig. 9 in which the maximum operating temperature of the strain gauges is intentionally exceeded may now be revisited and completed. The reader may recall that the results of Fig. 9 were obtained under experimental thermal conditions that destroyed the strain gauges on the side of the MFSP directly exposed to the hot platen. Now, with the finite element model, deformation data for the exterior side of the MFSP

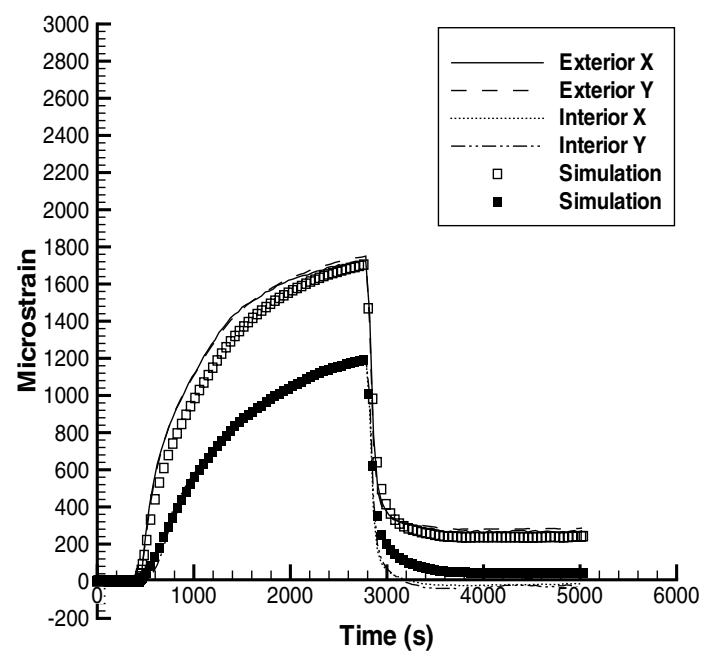

Fig. 11 Response of the MFSP with a platen temperature of $260^{\circ} \mathrm{C}$ $\left(500^{\circ} \mathrm{F}\right)$.

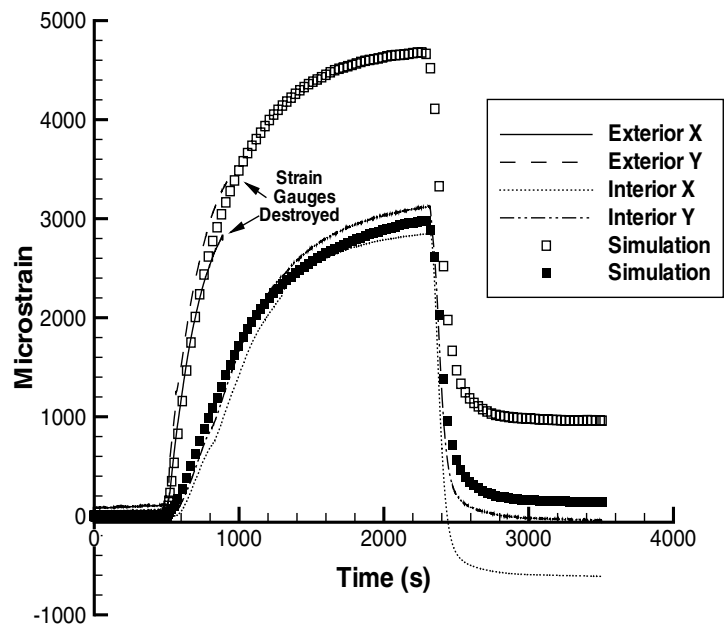

Fig. 12 Response of the MFSP with a platen temperature of $540^{\circ} \mathrm{C}$ $\left(\mathbf{1 0 0 4}^{\circ} \mathbf{F}\right)$.

may be obtained to complement the data obtained experimentally on the interior side of the panel. These results are shown in Fig. 12 .

\section{Conclusions}

The present work demonstrates and quantifies the effectiveness of actively cooled MFSPs in providing thermal protection while bearing in-plane loads. Inconel MFSPs are subjected to through-thethickness temperature gradients in a manner that simulates conditions to be expected in the primary fuselage panels of highspeed vehicles. In this sense, one side of the panel is considered the exterior of the vehicle and is directly subjected to elevated temperatures. The other side of the panel is considered the interior of the vehicle and is not subjected directly to the heat source. This investigation demonstrates that active cooling can eliminate elevated temperatures and thermomechanical deformation on the unheated side of an MFSP (i.e., the interior of the fuselage panel) for temperature exposure as high as $540^{\circ} \mathrm{C}\left(1004^{\circ} \mathrm{F}\right)$ on the exterior side of the panel. In addition, the thermomechanical deformation on the exterior side of the panel is greatly reduced via active cooling. It is reasonable to expect that, in test facilities capable of applying higher temperatures and greater coolant flow rates, such favorable performance could be demonstrated under more extreme thermal environments such as those that approach the thermal limits of the metallic structural material. [Inconel, with a melting point of $1300^{\circ} \mathrm{C}$ $\left(2372^{\circ} \mathrm{F}\right)$, performed successfully at temperatures of $700^{\circ} \mathrm{C}$ on the $X-15$.] In addition to demonstrating thermal protection, these results reveal the potential to control through active cooling the thermomechanical deformation of MFSPs.

\section{Appendix}

Before conducting a full thermostructural response experiment for the actively cooled MFSP, it is essential to acquire a quantitative understanding of the thermal performance of MFSPs so that system parameters may be chosen appropriately for the experimental specimen and procedure. The work addressed below is an investigation into the effects on the thermal performance of MFSPs by varying two primary system parameters: metal foam density and coolant flow rate.

\section{Experimental Procedure}

Five MFSPs are used in the study spanning a range of foam densities of $\rho_{f}=5.5,8.0,9.0,10.2$, and $12.2 \%$. The panels have the same cross-sectional geometry as the inconel MFSPs studied throughout the present paper, i.e., $6.4 \mathrm{~mm}(0.25 \mathrm{in}$.) core thickness with $1 \mathrm{~mm}$ (0.04 in.) face sheet thickness. The materials are 6061-T6 aluminum for the face sheets and 6101-T6 aluminum for the core. 
The panels measure $203.3 \mathrm{~mm}$ (8.0 in.) in length and $50.8 \mathrm{~mm}$ (2.0 in.) in width.

The experimental articles are tested in the setup depicted in Fig. A1. One side of the panel is heated by a $500 \mathrm{~W}$ heat lamp, and all other sides of the panel are free convection boundaries. The panel is fitted with a manifold so that compressed air may be passed through the foam core and freely out-gassed to the atmosphere. The airflow is monitored by an inline flow meter and pressure gauge. For all experiments discussed below, the heat lamp is run at a constant power setting and constant elevation, $170 \mathrm{~mm}$ (6.7 in.), above the top surface of the test articles. The temperature on the heated and unheated sides of the panel is captured with type-K thermocouples attached to an electronic ice point.

At the start of the experiment, the panel is at $24^{\circ} \mathrm{C}\left(75^{\circ} \mathrm{F}\right)$ and the heat lamp is turned on without any active cooling. The temperature on the two surfaces of the panel is recorded at $10 \mathrm{~s}$ intervals as the temperature rises. Once the temperature on the heated side reaches $110^{\circ} \mathrm{C}\left(230^{\circ} \mathrm{F}\right)$, the coolant flow is activated. The temperature on the panel surfaces is continually monitored until thermal equilibrium is achieved. This experiment is conducted on all five panel densities and for a variety of flow rates.

\section{Results and Discussion}

Figure A2 displays data recovered from one of these experiments, which serves as a good example of the type of results observed in each of the experiments. This particular experiment involved a panel with $\rho_{f}=8 \%$ and $\Delta P=20.7 \mathrm{kPa}$ (3 psi). The temperature in the uncooled panel rises similarly on both the heated and the unheated panel. Once the cooling is initiated, the temperature on each side of the panel drops dramatically and quickly establishes a new thermal equilibrium.

Of particular interest for actively cooled structural panels, in general, is the role of the metal foam core in the heat transfer capabilities of the structural thermal protection system. To illustrate this, compare the response displayed in Fig. A2 to that shown in Fig. A3 for an identical panel that has no foam core at all, only an air gap.

Qualitatively, the responses are very different. In the uncooled foamed panel, the temperature on both sides of the panel rises almost identically at an average rate of approximately $0.375^{\circ} \mathrm{C} / \mathrm{s}$ $\left(0.675^{\circ} \mathrm{F} / \mathrm{s}\right)$. In the uncooled hollow panel, the temperature on the heated side rises nearly twice as fast as that in the unfoamed panel, $0.72^{\circ} \mathrm{C} / \mathrm{s}\left(1.3^{\circ} \mathrm{F} / \mathrm{s}\right)$, whereas the temperature on the unheated side rises relatively slowly and moderately.

In the context of hot uncooled panels, the foamed panel actually provides a disadvantage in performance by assisting in the conduction of heat into the unheated side of the panel, which would be the interior of the vehicle. However, once cooled, the foamed panel provides distinct advantages seen in these plots. First, the foam assists in conducting heat from the heated surface of the MFSP into the cross section of the coolant flow. Second, with respect to the fact that MFSPs are being considered for a multifunctional solution to the problem of bearing airframe mechanical loads and thermal loads, the panel without a foam core is not a structurally viable component.

To design an actively cooled MFSP to meet its thermal protection requirement, it is necessary to identify the trends in the thermal performance of MFSPs due to foam density and coolant flow rates.

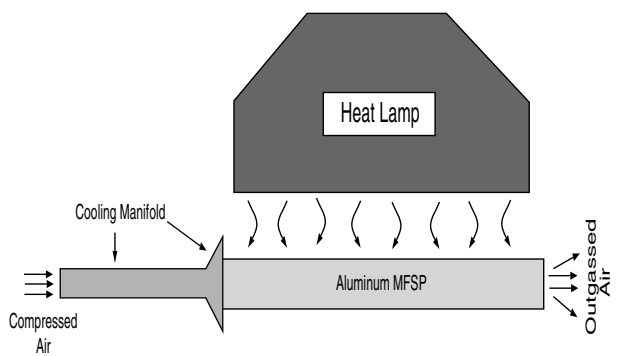

Fig. A1 Setup for the parametric study on the thermal performance of MFSPs.

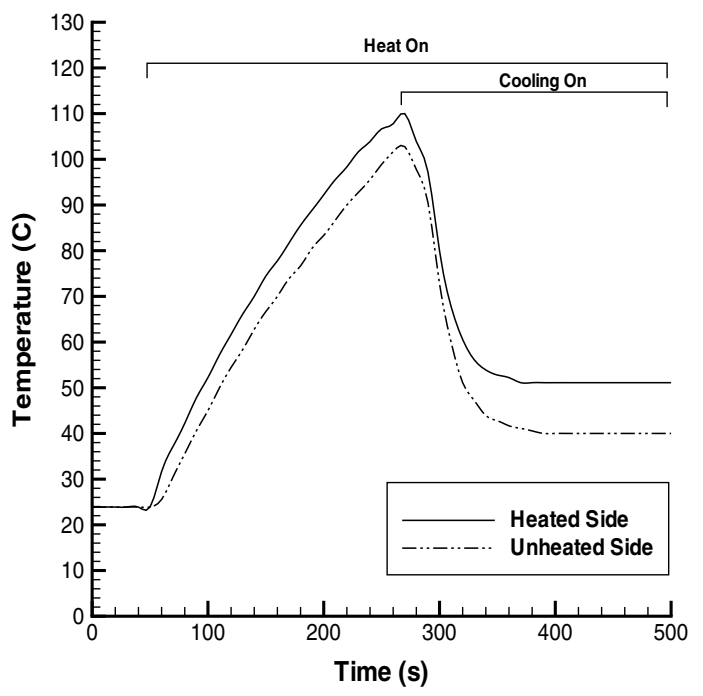

Fig. A2 Sample plot of the thermal response of an actively cooled MFSP.

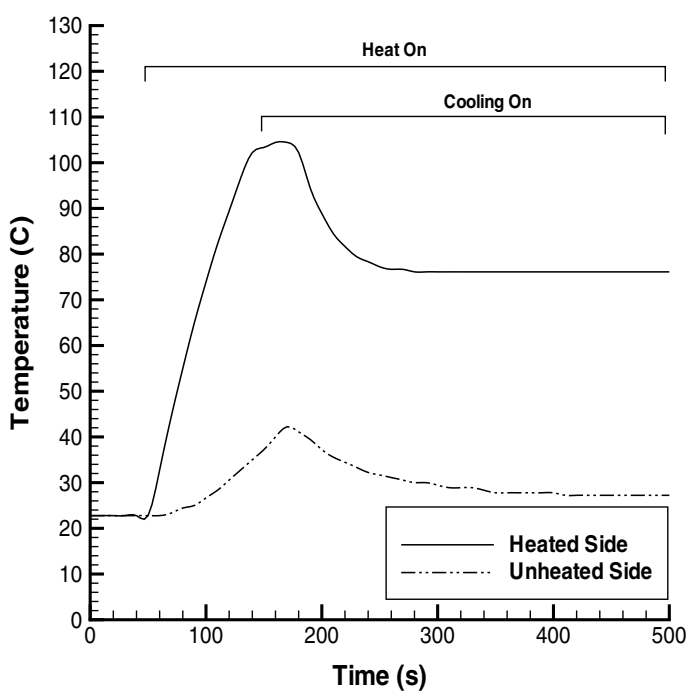

Fig. A3 Effect of removing the foam core.

An experimental investigation into this issue showed that foam density, within the range investigated, does not have an appreciable or clearly defined effect on the thermal performance of MFSPs. So as not to waste effort tailoring thermal performance via foam density, it is valuable to identify that foam density is not a major consideration in the thermal performance of MFSPs and may, therefore, be a variable solely defined by mechanical performance requirements.

Differing pressure drops are required to establish identical flow rates in panels of different foam densities. Thus, instead of examining the effect of flow rate on the thermal performance of MFSPs, the effect of pressure drop is investigated. An experimental investigation into this issue identified that once $\Delta P 30 \geq \mathrm{kPa}(4.4 \mathrm{psi})$, an increase in pressure drop has only a marginal effect on decreasing the panel temperatures. Thus, any pressure drop greater than this value should be appropriate in the thermomechanical experiments. It was found that room temperature, $24^{\circ} \mathrm{C}\left(75^{\circ} \mathrm{F}\right)$, can be maintained on the unheated side of the panel if $P=70 \mathrm{kPa}(10.1 \mathrm{psi})$.

\section{Acknowledgements}

This work was supported by the Space Vehicle Technology Institute under Grant No. NCC3-989, jointly funded by NASA and the Department of Defense within the NASA Constellation University Institutes Project, with Claudia Meyer as the project manager. The authors are grateful to Yanyu Luo, Ken Salas, and 
Kristen Montz for their efforts in conducting the experimental work presented in the appendix.

\section{References}

[1] Kelly, H. N., and Blosser, M. L., "Active Cooling from the Sixties to NASP," Current Technology for Thermal Protection Systems, CP3157, NASA, 1992.

[2] Ellis, D. A., Pagel, L. L., and Schaeffer, D. M., "Design and Fabrication of a Radiative Actively Cooled Honeycomb Sandwich Structural Panel for Hypersonic Aircraft," NASA CR-2957, 1978.

[3] Evans, A. G., Hutchinson, J. W., Ashby, M. F., "Multifunctionality of Cellular Metal Systems," Progress in Materials Science, Vol. 43, No. 3 , 1998, pp. 171-221.

[4] Rakow, J. F., and Waas, A. M., "Size Effects and the Shear Response of Aluminum Foam," Mechanics of Materials, Vol. 37, No. 1, 2004, pp. 297-310.

[5] Rakow, J. F., and Waas, A. M., "Size Effects in Metal Foam Cores for Sandwich Structures," AIAA Journal, Vol. 42, No. 7, 2004, pp. 13311337.

[6] Rakow, J. F., and Waas, A. M., "The Elastic Moduli of Random Fibrous Composites, Platelet Composites, and Foamed Solids," Mechanics of Advanced Materials and Structures, Vol. 11, No. 2, 2004, pp. 151-173.
[7] Incropera, F. P., and DeWitt, D. P., Fundamentals of Heat and Mass Transfer, Wiley, New York, 2002, pp. 364-367.

[8] Rakow, J. F., and Waas, A. M., "Thermal Buckling of Metal Foam Sandwich Panels for Actively Cooled Thermal Protection Systems," Journal of Spacecraft and Rockets, Vol. 42, No. 5, 2005, pp. 832-844.

[9] Rakow, J. F., "Thermomechanical Response of Metal Foam Sandwich Panels for Structural Thermal Protection Systems in Hypersonic Vehicles," Ph.D. Dissertation, University of Michigan, Ann Arbor, MI, 2005.

[10] Myers, D. E., Martin, C. J., and Blosser, M. L., "Parametric Weight Comparison of Advanced Metallic, Ceramic Tile, and Ceramic Blanket Thermal Protection Systems," NASA TM-2000-210289, June 2000

[11] Moosbrugger, C., Experimental Stress Analysis, ASM International, Materials Park, OH, 2002.

[12] ABAQUS, Ver. 6.4, ABAQUS, Inc., Warwick, RI. 2003.

[13] Dally, J. W., and Riley, W. F., Atlas of Stress-Strain Curves, McGrawHill, New York, 1978.

[14] Ashby, M. F., Evans, A. G., Fleck, N. A., Gibson, L. J., Hutchinson, J. W., and Wadley, H. N. G., Metal Foams: A Design Guide, ButterworthHeinemann, Boston, 2000.

A. Palazotto Associate Editor 